\title{
Live Demonstration: Real-Time Audio Restoration using Sparse Signal Recovery
}

\author{
D. Bellasi ${ }^{1}$, P. Maechler ${ }^{1}$, A. Burg ${ }^{2}$, N. Felber ${ }^{1}$, H. Kaeslin ${ }^{1}$, and C. Studer ${ }^{3}$ \\ ${ }^{1}$ ETH Zürich, Switzerland; e-mail: bellasid@ee.ethz.ch, \{maechler, felber, kaeslin\}@iis.ee.ethz.ch \\ ${ }^{2}$ EPFL Lausanne, Switzerland; e-mail: andreas.burg@epfl.ch \\ ${ }^{3}$ Rice University, USA; e-mail: studer@ rice.edu
}

\begin{abstract}
We demonstrate the restoration of audio signals corrupted by clicks and pops using techniques from sparse signal recovery and compressive sensing. The demonstration features real-time signal restoration using the approximate message passing algorithm on an FPGA prototyping board. To highlight the restoration performance of our implementation, we remove clicks and pops from old phonograph recordings in real time.
\end{abstract}

\section{INTRODUCTION}

Compressive sensing (CS) enables the acquisition and recovery of sparse signals using fewer measurements than the Nyquist rate suggests [1]. The main idea of CS, i.e., the recovery of sparse signals from a set of underdetermined linear measurements, has recently been applied to the restoration of sparsely corrupted signals, such as audio signals, images, and videos [2]. However, the algorithms required to perform sparse signal recovery typically exhibit very high computational complexity, which necessitates the development of novel efficient methods and the design of dedicated and high-performance hardware implementations.

\section{SPARsity-BASED SignAl RESTORATION}

The principles of CS can be used for the recovery of sparsely corrupted signals [2]. Specifically, by modeling the corrupted (and observed) signal $\mathbf{z} \in \mathbb{R}^{M}$ as $\mathbf{z}=\mathbf{A a}+\mathbf{B b}$, with the matrix $\mathbf{A} \in \mathbb{R}^{M \times N_{a}}$ used to sparsify the signal $\mathbf{s}=\mathbf{A a}$ to be restored, the matrix $\mathbf{B} \in \mathbb{R}^{M \times N_{b}}$ used to sparsify the (unwanted) corruptions, such as clicks, pops, and saturation artifacts. In order to perform recovery of the sparse representations $\hat{\mathbf{x}}=[\hat{\mathbf{a}} ; \hat{\mathbf{b}}]$ from $\mathbf{z}$, an appropriate pair of matrices $\mathbf{A}$ and $\mathbf{B}$ is required [2]. For audio-signal restoration, we set $\mathbf{A}$ to the $M \times M$ discrete cosine transform (DCT) matrix to sparsify audio signals, and $\mathbf{B}$ to the identity basis, which sparsifies clicks, pops, and saturation artifacts. Audio-signal restoration now amounts to recovering the sparse representations $\hat{\mathbf{x}}=[\hat{\mathbf{a}} ; \hat{\mathbf{b}}]$ from $\mathbf{z}$ with the aid of a sparse signal recovery algorithm, followed by computing an estimate of the uncorrupted signal via $\hat{\mathbf{s}}=\mathbf{A} \hat{\mathbf{a}}$.

The sparse signal recovery algorithm of choice is the approximate message passing (AMP) algorithm [3], which exhibits low computational complexity and enables the efficient implementation in VLSI [4]. The proposed VLSI architecture is capable to perform real-time audio restoration at low hardware complexity, by using a high-throughput architecture of the DCT relying on an efficient FFT implementation [4].

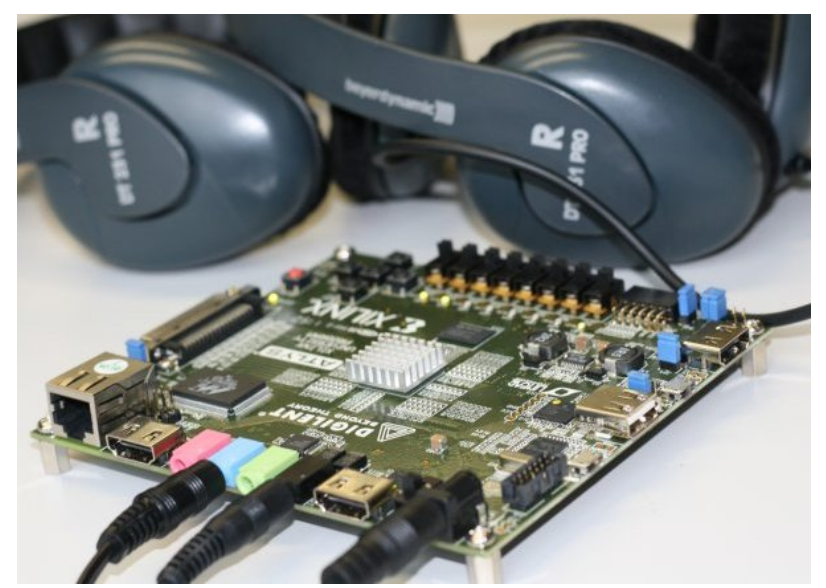

Fig. 1. Demonstration setup with the FPGA prototyping board.

\section{LIVE DEMONSTRATION}

The live demonstration highlights the restoration capabilities of the proposed CS-based recovery framework for sparsely corrupted 16 bit $44.1 \mathrm{kHz}$ stereo audio signals. Restoration is performed in real time on a Xilinx Spartan-6 XC6SLX45-3 FPGA residing on a Digilent Atlys prototyping board (see Fig. 1) using an efficient implementation of the fast DCT-based AMP architecture together with an AC'97 audio interface.

This live demonstration gives the audience an impression of the performance of a sparsity-based signal restoration by listening to a variety of corrupted audio signals, e.g., old phonograph recordings. The proposed AMP implementation performs real-time audio restoration on the FPGA prototyping board and the involved algorithm parameters can be modified at run-time, which enables one to explore the capabilities of signal restoration using techniques from compressive sensing.

\section{REFERENCES}

[1] E. Candès and M. Wakin, "An introduction to compressive sampling," IEEE Signal Process. Mag., vol. 25, no. 2, pp. 21-30, March 2008.

[2] C. Studer and R. G. Baraniuk, "Stable restoration and separation of approximately sparse signals," submitted to Appl. Comput. Harmon. Anal., ArXiv preprint: 1107.0420.

[3] D. Donoho, A. Maleki, and A. Montanari, "Message-passing algorithms for compressed sensing," Proc. of the National Academy of Sciences, vol. 106, no. 45, pp. 18914-18919, Sept. 2009.

[4] P. Maechler et al., "VLSI design of approximate message passing for signal restoration and compressive sensing," IEEE J. on Emerging and Sel. Topics in Circuits and Systems, vol. 2, no. 3, 2012. [Online]. Available: www.iis.ee.ethz.ch/ maechler/papers/amp12.pdf 